polygraph record is merely a continuation of Strip A, after 3 sec.

During the first portion of Strip A, the drinkometer had been turned off electrically. This is indicated by the absence of deflections in the second tracing. During this portion of the record, it can be seen that the EKG was stable and artifact-free and that licking responses were clearly indicated by the amplified output of the crystal. ${ }^{3}$ Midway through Strip A, the drinkometer was turned on. This event is indicated by the occurrence of deflections in the signal marker, which are perfectly correlated with the peaks in the record corresponding to the output of the crystal. At the same time, however, artifacts become apparent in the EKG tracing. The drinkometer remained on for approximately $6 \mathrm{sec}$, until a point midway through Strip B. When the drinkometer was turned off, the artifacts disappeared and within $1 \frac{1}{2} \mathrm{sec}$ the EKG was again readable and at the original base line. ${ }^{4}$ It is concluded, therefore, that the method for monitoring drinking behavior presented in this paper has all of the essential features of the original method (Hill \& Stellar, 1951) plus the important additional advantage of being compatible with procedures for recording electrophysiological phenomena.

\section{REFERENCES}

EISMAN, E. Technique for monitoring cardiac function without handling or retraining the animal. Perceptual \& Motor Skills, 1965, 20, 1093-1097.

HILL, J. H., \& STELLAR, E. An electronic drinkometer. Science, 1951, $114,43-44$.

\section{NOTES}

1. The work in this paper was supported by Grant No. 162-7442 from the Graduate School of the University of Wisconsin.

2. A Grass Model 5 polygraph, with a Model 5P1 preamplifier, was used to record the output of the crystal. The selector switch was set for a time constant of $.1 \mathrm{sec}$ and the high-frequency filter was set at 60 . The gain settings were such that a deflection of $1 \mathrm{~cm}=100 \mathrm{mV}$. The chart paper shown in Fig. 1 was printed with a spacing of $5 \mathrm{~mm}$ per box.

3. For digital measurements, a commercially available audio detection relay (Scientific Prototype Co.) has been used, with good success. The output of the crystal is fed directly to the input of the relay, with no preamplification required. Cumulative recorders and electromechanical counters can then be operated, as in the Hill-Stellar method.

4. The relatively long period of time required for restoration of the EKC trace is tentatively attributed to a property of the amplifier in the polygraph rather than to a persistent aftereffect, within the animal, of the passage of current from the drinkometer.

\title{
Technique for monitoring activity in free-moving animals
}

\author{
E U G E N E E I S M A N, UNIVERSITY \\ WISCONSIN-MIL WAUKEE, Milwaukee, Wisconsin 53201
}

$O F$

A techique is described for measuring activity by monitoring the rotational movements of a commutator, to which animals might be connected for other experimental purposes.

A method is presented in this paper for measuring behavioral activity in the rat. The technique is particularly appropriate for use with animals that would otherwise be prepared for electrophysiological recording or stimulation. In such preparations, overhead leads are usually carried from a terminal on the animal's head to a commutator situated above the experimental cage. It is the feature of the commutator that provides the means for measuring activity in this method.

A typical commutator is constructed of a circular disk with two concentric depressions to contain mercury. The disk rotates freely on a stationary vertical post through its center. Lead wires from the animal are connected to terminals on the underside of the disk, and through them, to the mercury contained in the depressions. A square cover is positioned above the disk, with the post through its center. Stationary electrodes project downward through the cover and into the mercury, to complete the circuit through the commutator. When animals are connected to the commutator, using properly counterweighted leads (Eisman, 1965), it is observed that even small movements are faithfully reflected in rotary movements of the disk. Activity can be measured by monitoring these rotary movements. The latter is accomplished by mounting a magnetic reed switch along one edge of the square cover and a small cylindrical magnet on the circumference of the disk. The long axes of the magnet, and the switch, are arranged parallel to the plane of rotation of the commutator. The switch is activated, therefore, each time the magnet passes beneath its center-point. Each closure of the switch can then be monitored on an electromagnetic counter, a

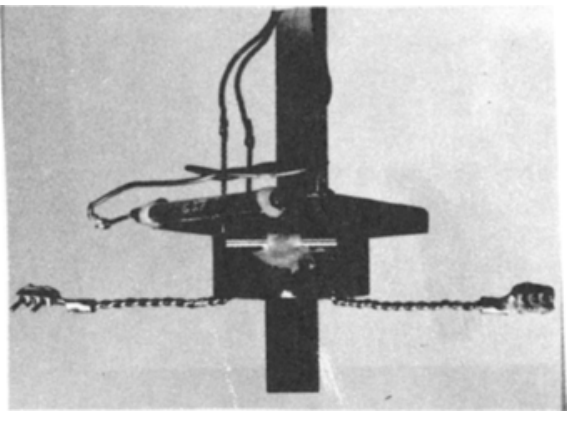

Fig. 1. A simple commutator with a reed switch mounted on the rotating disk and a magnet on the cover.

cumulative recorder, or the like, to obtain the measure of activity.

The sensitivity of the commutator system can be varied by using a configuration involving more than one reed switch. For example, it is possible to mount two switches, on opposite edges of the cover. Electrical circuitry can then be arranged so that a count is obtained from one of the switches, but only after the other switch has been closed at least once. Thus, if an animal were initially facing the front of the cage, it would have to turn toward the rear, and then in either direction back to the front, in order for a movement to be recorded. In this manner, it is possible to detect quite minor movements of the $\mathrm{S}$ or only those movements that yield some criterial degree of rotation in the commutator.

An application of the present method is illustrated in Fig. 2. A commutator with one reed switch, and a cumulative recorder were used to monitor the activity of a free-moving rat under two different temperature conditions. The animal was housed in an 
A

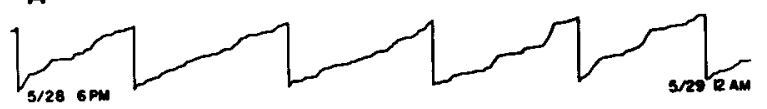

$\mathbf{B}$

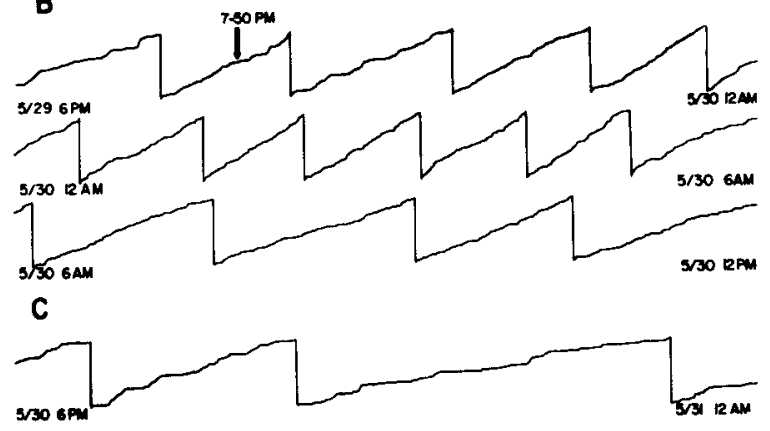

Fig 2. Activity as a function of ambient temperature. The change in temperature, from $40 \mathrm{deg} F$ to $90 \mathrm{deg} F$, is indicated by the vertical arrow.

experimental cage, located inside an environmental chamber, and had previously been prepared for cardiac recording, by methods described elsewhere (Eisman, 1965). The cage measured $6 \times 12 \times 20$ in. $\mathrm{H}$, and had the commutator centered $15 \mathrm{in.}$ above the top. The animal remained in the cage, with its leads continuously attached to the commutator, for a period of 30 days, including the time shown in Fig. 2. During the entire period, ambient temperature had been altered a number of times. For the previous 3 days, and until the time indicated by the arrow in Fig. 2, it had been maintained at $40 \mathrm{deg} F$, and was then shifted to $90 \mathrm{deg} F$. This transition required less than $15 \mathrm{~min}$, by virtue of an efficient heating system in the chamber.

The records shown in Fig. 2 clearly indicate that the shift in temperature was followed by a change in activity. Each horizontal strip represents a 6-h period, and each complete vertical excursion of the pen required 300 closures of the switch.
Panels A, B, and C begin at the same hour, respectively, on the day of, and the day after, the shift in temperature. Two additional strips were included in Panel $B$, to provide a more detailed record in the period following the change. It can be seen that there was a marked increase in the rate of activity during the $10 \mathrm{~h}$ following the change in temperature. The rate then began to decline and by the following day, it was appreciably below the original level. In approximate numerical values, estimated from the cumulative record, there were 1,575 counts on the day before the change and 800 counts on the day after, which represents a decrease of $49 \%$.

The generality of the findings obtained by the present method, and therefore its utility, might be indicated by comparing the latter numerical value with one obtained in a more familiar method. Stevenson and Rixon (1957), for example, measured activity in a running wheel under comparable temperature levels and obtained counts of 700 and $450 \mathrm{rev} /$ day, respectively. This represents a decrease of $36 \%$. In view of the differences in procedure, the length of the observational periods, and the number of animals involved, the two values are considered sufficiently close to suggest that the new method is sensitive to at least some of the same underlying phenomena as are measured in the running wheel. Accordingly, the commutator system is offered as a useful technique for measuring activity, especially in situations that would otherwise employ overhead leads, for any purpose. Under such circumstances, the new method provides an obvious advantage in simplicity, and perhaps in reliability and sensitivity, over the usual methods involving floor-activated switches, photobeams, or contact relays.

\section{REFERENCES}

EISMAN, E. Technique for monitoring cardiac function without handling or restraining the animal. Perceptual \& Motor Skills, 1965, 20, 1093-1097.

STEVENSON, J. A. F., \& RIXON, R. H. Environmental temperature and deprivation of food and water on the spontaneous activity of rats. Yale Joumal of Biological Medicine, 1957, 29, 575-584.

\section{Note}

1. The work of this paper was supported by Grant No. 162-7442 from the Graduate School of the University of Wisconsin. 